\title{
PCx User Guide
}

by

Joseph Czyzyk, Sanjay Mehrotra, and Stephen J. Wright

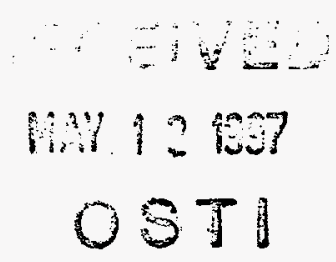

-

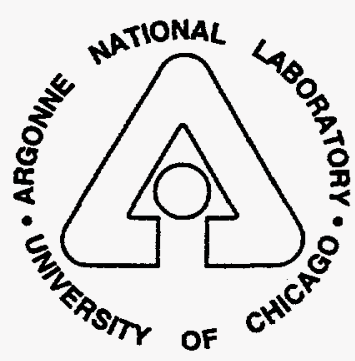

in

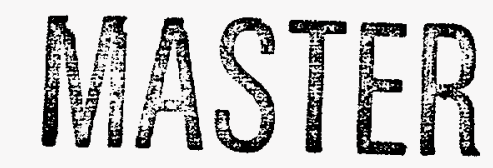

MATHEMATICS AND
COMPUTER SCIENCE

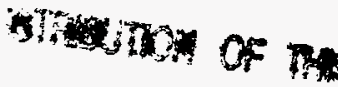




\title{
ARGONNE NATIONAL LABORATORY
}

9700 South Cass Avenue

Argonne, IL 60439

ANL/MCS-TM-217

\section{PCx User Guide}

\author{
by \\ Joseph Czyzyk, Sanjay Mehrotra, and Stephen J. Wright
}

Mathematics and Computer Science Division

Technical Memorandum No. 217

March 1997

This work was supported by the Mathematical, Information, and Computational Sciences Division subprogram of the Office of Computational and Technology Research, U.S. Department of Energy, under Contract W-31-109-Eng38. 


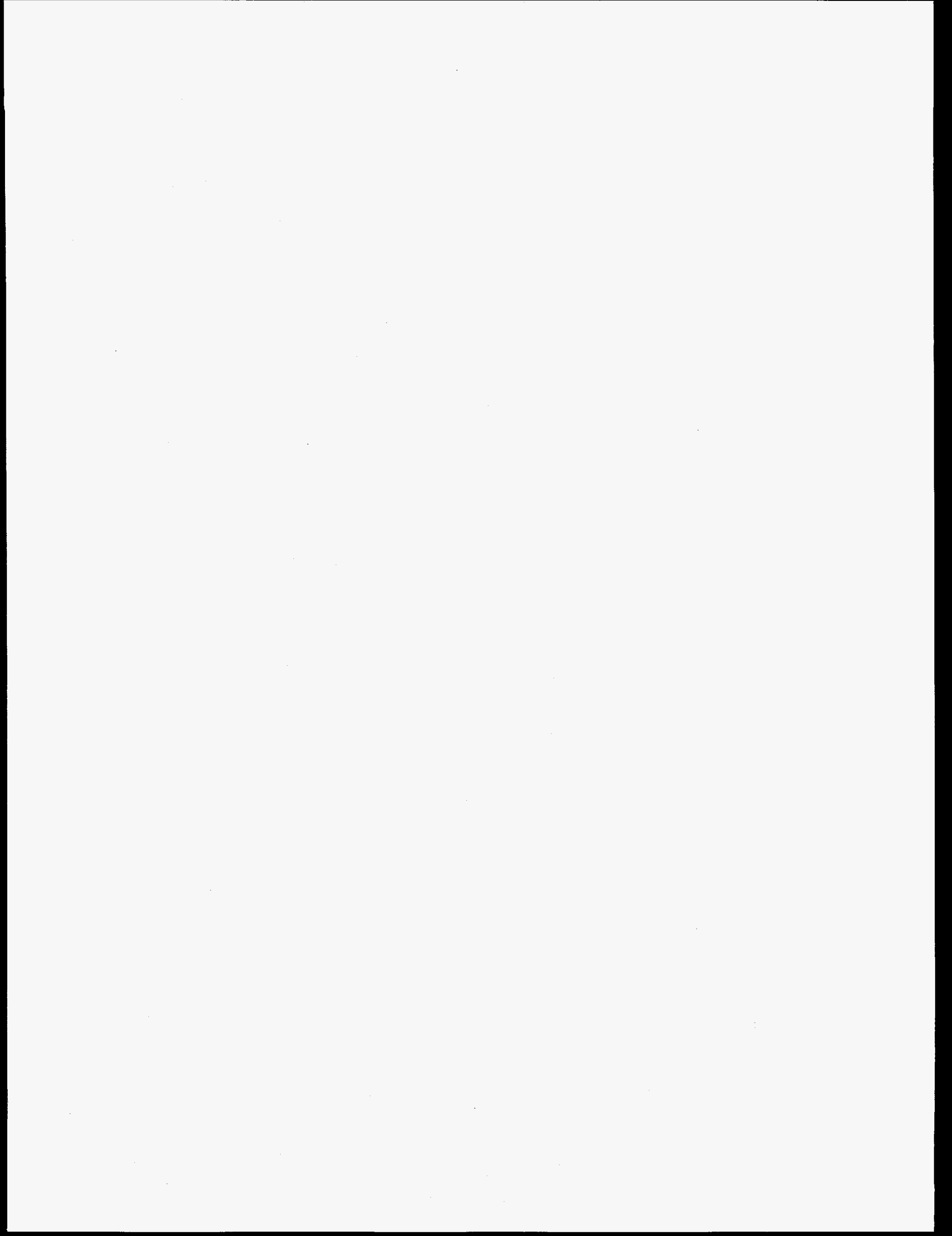




\section{Contents}

$\begin{array}{ll}\text { Abstract } & 1\end{array}$

1 Introduction 1

2 The Formulation $\quad 2$

3 The Algorithm $\quad 4$

4 Linear Algebra $\quad 7$

5 The Presolver $\quad 10$

6 Specifications File $\quad 11$

7 Obtaining and Installing PCx 13

8 Invoking $\mathrm{PCx} \quad 15$

9 Computational Results $\quad 15$

$\begin{array}{lr}\text { Acknowledgments } & 20\end{array}$

$\begin{array}{lc}\text { References } & \mathbf{2 0}\end{array}$ 


\title{
PCx User Guide
}

\author{
by \\ Joseph Czyzyk, Sanjay Mehrotra, and Stephen J. Wright
}

\begin{abstract}
We describe the code PCx, a primal-dual interior-point code for linear programming. Information is given about problem formulation and the underlying algorithm, along with instructions for installing, invoking, and using the code. Computational results on standard test problems are tabulated. The current version number is 1.0 .
\end{abstract}

Key words: linear programming, interior-point methods, software.

\section{Introduction}

PCx is a linear programming solver developed at the Optimization Technology Center at Argonne National Laboratory and Northwestern University. It implements a variant of Mehrotra's predictorcorrector algorithm [7] with the higher-order correction strategy of Gondzio [4]. This primal-dual approach has proved to be the most efficient interior-point method for general linear programs. The bulk of $\mathrm{PCx}$ is written in the $\mathrm{C}$ programming language. However, its main computational engine-the sparse Cholesky code of $\mathrm{Ng}$ and Peyton [8]-is coded in Fortran 77. Source code for both PCx and Ng and Peyton's solver is available subject to the qualifications in the copyright statement on the PCx home page on the World Wide Web (see Section 7).

Key features of PCx include

- a set of high-level data structures for linear programming constructs, designed for possible reuse in other codes;

- ability to be invoked both as a stand-alone program (with input from an MPS file) and as a callable procedure;

- a presolver;

- modular structure, which makes it easy for users to modify the code to experiment with variants of the current algorithm.

The current version of $\mathrm{PCx}$ performs efficiently on the standard netlib test problems. Nevertheless, PCx should be viewed as work in progress. Features such as finite termination/basis recovery and alternative linear algebra solvers (and alternative formulations of the step equations) may be added in future versions. In making the source available, we encourage others to become involved in the development and extension of $\mathrm{PCx}$.

The remaining sections of this guide contain an outline of the underlying algorithm, instructions for installing and using $\mathrm{PCx}$, and computational results on standard test problems. Section 2 describes the various linear programming formulations that are accommodated by the data structures of $\mathrm{PCx}$, including the formulation to which the algorithm is actually applied. Section 3 describes the algorithm, including details of termination and infeasibility detection. Section 4 
discusses the major computational issue in the code-factorization of a sparse, positive definite matrix-including the modifications to the $\mathrm{Ng}$-Peyton code [8] needed in this context. Presolver capabilities are outlined in Section 5 . Section 7 contains instructions for installing the code in a Unix environment, while instructions for invoking $\mathrm{PCx}$ as a stand-alone solver are given in Section 8. The user can set various algorithmic options and control the amount and type of output by means of a specifications file; details are provided in Section 6 . Finally, Section 9 reports on computational results for the standard netlib test set of feasible and infeasible problems, together with some new problems arising from the NEMS project at Argonne.

This guide will be updated continually as new releases of the code are made.

\section{The Formulation}

PCx accepts any valid linear program that can be specified in the MPS format. The model described in the MPS file may include upper and lower bounds, linear equality constraints, linear inequality constraints and free variables. PCx defines a data structure MPStype that contains a complete specification of a single linear programming problem in this general formulation. This data structure also stores the names assigned to the rows, columns, and objectives of the model specified in the MPS file.

For algorithmic purposes, however, it is convenient to work with a simpler formulation of the linear program. PCx converts the general formulation to the following simpler form:

$$
\begin{array}{lll}
\min _{x \in R^{n}} c^{T} x \text { subject to } A x=b & 0 \leq x_{i}, & i \in \mathcal{N} \\
& 0 \leq x_{i} \leq u_{i}, \quad i \in \mathcal{U} \\
& x_{i} \text { free, } & i \in \mathcal{F},
\end{array}
$$

where $\mathcal{N} \cup \mathcal{U} \cup \mathcal{F}$ is a partition of the index set $\{1,2, \ldots, n\}$ into "normal," "upper-bounded," and "free" variables, respectively. The PCx data structure LPtype contains a single linear program in the form (1). The transformation from an MPStype formulation to an LPtype formulation is carried out internally and transparently to the user by PCx. After the solution has been found, the transformation from MPStype to LPtype is inverted to express the solution in terms of the original formulation.

Users who circumvent the MPS file and call the procedure $\mathrm{PCx}()$ directly must specify their problems in the form (1). That is, they pass an LPtype data structure to this procedure.

The current version of PCx carries out one more level of problem transformation before invoking the solution algorithm. The use of a normal equations formulation of the step equations (see below) implies that the model can contain no free variables. Hence, we replace each of the free variables $x_{i}$ in the LPtype formulation by a pair of normal variables $x_{i}^{+}$and $x_{i}^{-}$, making the substitution

$$
x_{i}=x_{i}^{+}-x_{i}^{-} \text {. }
$$

After these substitutions are made (and the notation is redefined), the linear program has the following form:

$$
\begin{array}{lll}
\min _{x \in R^{n}} c^{T} x \text { subject to } A x=b & 0 \leq x_{i} \leq u_{i}, & i \in \mathcal{U} \\
& 0 \leq x_{i}, & i \in \overline{\mathcal{U}},
\end{array}
$$

where $\overline{\mathcal{U}}=\{1,2, \ldots, n\} \backslash \mathcal{U}$. The split variables are recombined before return from PCx(), so the transformation between (1) and (2) is transparent to the user. The LPtype data structure is also used to store problems in the form (2). 
The dual problem associated with (2) is

$$
\begin{aligned}
\max _{\pi \in R^{m}, r \in R^{|\mathcal{U}|}, s \in R^{n}} b^{T} \pi-\sum_{i \in \mathcal{U}} u_{i} r_{i} & \\
\text { subject to } \quad A_{i}^{T} \pi+s_{i}-r_{i} & =c_{i} \quad i \in \mathcal{U} \\
A_{i \cdot \pi}^{T}+s_{i} & =c_{i} \quad i \in \overline{\mathcal{U}} \\
(r, s) & \geq 0,
\end{aligned}
$$

where $\pi$ is the Lagrange multiplier vector for the equality constraint $A x=b$, and $r$ represents the Lagrange multipliers for the upper bounds $x_{i} \leq u_{i}$. The Karush-Kuhn-Tucker (KKT) optimality conditions for (2) and (3) are

$$
\begin{array}{rlrl}
A_{i \cdot \pi}^{T} \pi+s_{i}-r_{i} & =c_{i}, & i \in \mathcal{U} \\
A_{i}^{T} \pi+s_{i} & =c_{i}, & i \in \overline{\mathcal{U}} \\
A x & =b & \\
x_{i}+w_{i} & =u_{i}, & i \in \mathcal{U}, \\
x_{i} s_{i} & =0, \quad i=1,2, \ldots, n, \\
w_{i} r_{i} & =0, \quad i \in \mathcal{U}, \\
(x, s, r, w) & \geq 0 .
\end{array}
$$

(We have introduced a vector $w$ of slack variables for the constraint $x_{i} \leq u_{i}$.)

Like all infeasible-primal-dual algorithms, the version of Mehrotra's algorithm implemented by $P C x$ generates a sequence of iterates

$$
\left(x^{k}, \pi^{k}, s^{k}, r^{k}, w^{k}\right), \quad k=0,1,2, \ldots,
$$

that satisfy the strict positivity condition $\left(x^{k}, s^{k}, r^{k}, w^{k}\right)>0$. However, these points are usually infeasible; that is, the equality conditions (4a), (4b), (4c) are satisfied only in the limit as $k \rightarrow \infty$. Compliance with the complementarity conditions $(4 \mathrm{e}),(4 \mathrm{f})$ is measured by the duality measure $\mu$, defined by

$$
\mu=\frac{\sum_{i=1, \ldots, n} x_{i} s_{i}+\sum_{i \in U} w_{i} r_{i}}{n+|\mathcal{U}|}
$$

Note that $\mu$ is the average value of all the pairwise products $x_{i} s_{i}, i=1,2, \ldots, n$, and $r_{i} w_{i}, i \in \mathcal{U}$.

For simplicity in describing the algorithm, we assume in the remainder of the paper that all primal variables have upper bounds, that is, $\mathcal{U}=\{1,2, \ldots, n\}$. The primal and dual problems can be stated in this case as

$$
\min _{x \in R^{n}} c^{T} x \quad \text { subject to } \quad A x=b, 0 \leq x \leq u,
$$

and

$$
\max _{\pi \in R^{m}, r \in R^{n}, s \in R^{n}} b^{T} \pi-r^{T} u \quad \text { subject to } \quad A^{T} \pi+s-r=c, \quad(r, s) \geq 0 .
$$

The KKT conditions for (6) and (7) are

$$
A \pi+s-r=c,
$$




$$
\begin{aligned}
A x & =b, \\
x+w & =u, \\
x_{i} s_{i} & =0, \quad i=1,2, \ldots, n, \\
w_{i} r_{i} & =0, \quad i=1,2, \ldots, n, \\
(x, s, r, w) & \geq 0 .
\end{aligned}
$$

We stress that the PCx code actually works with the formulation (2); we use the simpler form (6) in our discussion solely to avoid creating a notational jungle in the next few sections.

\section{The Algorithm}

Mehrotra's predictor-corrector algorithm [7] is based on Newton's method for the KKT conditions (4a)-(4e), modified to retain positivity of the $(x, s, r, w)$ components, to incorporate a "centering" component in the search direction, and to improve the order of accuracy to which the search direction approximates the nonlinear equations (4d) and (4e). We mention just the major elements of the algorithm in this section and the next. For further details and motivation, see Wright [10].

The search direction at each iteration of Mehrotra's algorithm is obtained by solving two systems of linear equations, which have the same coefficient matrix but different right-hand sides. If we assume for simplicity that $\mathcal{U}$ in (2) is the entire index set $\{1,2, \ldots, n\}$, these step equations have the form

$$
\left[\begin{array}{ccccc}
0 & A & 0 & 0 & 0 \\
A^{T} & 0 & I & 0 & -I \\
0 & I & 0 & I & 0 \\
0 & S & X & 0 & 0 \\
0 & 0 & 0 & R & W
\end{array}\right]\left[\begin{array}{c}
\Delta \pi \\
\Delta x \\
\Delta s \\
\Delta w \\
\Delta r
\end{array}\right]=\left[\begin{array}{c}
-r_{b} \\
-r_{c} \\
-r_{u} \\
-r_{x s} \\
-r_{w r}
\end{array}\right] .
$$

Here $A$ is the constraint matrix from the linear program, $X=\operatorname{diag}(x), S=\operatorname{diag}(s), W=\operatorname{diag}(w)$, and $R=\operatorname{diag}(r)$. The coefficient matrix is simply the Jacobian of the nonlinear equations defined by (4a)-(4e). The right-hand side for the first system of equations chooses $r_{u}, r_{c}$, and $r_{b}$ to be the residuals for the upper-bound, dual, and primal infeasibilities, respectively; that is,

$$
r_{u}=x+w-u, \quad r_{c}=A^{T} \pi+s-r-c, \quad r_{b}=A x-b .
$$

For the other right-hand side components, this first system uses

$$
r_{x s}=X S e, \quad r_{r w}=R W e,
$$

so that the solution ( $\Delta x^{\text {aff }}, \Delta \pi^{\text {aff }}, \Delta s^{\text {aff }}, \Delta r^{\text {aff }}, \Delta w^{\text {aff }}$ ) of this first system is the pure Newton direction for the nonlinear system of equations (4a)-(4e). This direction is often known as the affine-scaling direction.

The second direction is a combined centering-corrector direction, which we denote by

$$
\left(\Delta x^{\mathrm{cc}}, \Delta \pi^{\mathrm{cc}}, \Delta s^{\mathrm{cc}}, \Delta r^{\mathrm{cc}}, \Delta w^{\mathrm{cc}}\right) \text {. }
$$

To obtain this direction, we set the right-hand side components of (9) as follows:

$$
\begin{gathered}
r_{u}=0, \quad r_{c}=0, \quad r_{b}=0, \\
r_{x s}=\Delta X^{\mathrm{aff}} \Delta S^{\mathrm{aff}} e-\sigma \mu e, \quad r_{r w}=\Delta R^{\mathrm{aff}} \Delta W^{\mathrm{aff}} e-\sigma \mu e,
\end{gathered}
$$


where $\mu$ is defined in (5) and $\Delta X^{\text {aff }}, \Delta S^{\text {aff }}, \Delta R^{\text {aff }}$, and $\Delta W^{\text {aff }}$ are the diagonal matrices constructed from the affine-scaling step components $\Delta x^{\text {aff }}, \Delta s^{\text {aff }}, \Delta r^{\text {aff }}$, and $\Delta w^{\text {aff }}$, respectively. The scalar $\sigma \in[0,1]$ in (12) is chosen by a complicated heuristic that is based on the ability of the pure affine-scaling step to attain large reductions in the duality measure $\mu$ before reaching the boundary of the positive orthant for the $(x, s, r, w)$ components. Given the affine-scaling step, we calculate the maximum step to this boundary in primal and dual variables from the definitions

$$
\begin{aligned}
\alpha^{\text {aff }, \mathrm{P}} & =\inf \left\{\alpha \in[0,1] \mid(x, w)+\alpha\left(\Delta x^{\text {aff }}, \Delta w^{\text {aff }}\right) \geq 0\right\}, \\
\alpha^{\text {aff,D }} & =\inf \left\{\alpha \in[0,1] \mid(s, r)+\alpha\left(\Delta s^{\text {aff }}, \Delta r^{\text {aff }}\right) \geq 0\right\} .
\end{aligned}
$$

We then compute the duality measure $\mu^{\text {aff }}$ at this point as

$$
\mu^{\mathrm{aff}}=\frac{1}{2 n}\left[\left(x+\alpha^{\mathrm{aff}, \mathrm{P}} \Delta x\right)^{T}\left(s+\alpha^{\mathrm{aff}, \mathrm{D}} \Delta s\right)+\left(w+\alpha^{\mathrm{aff}, \mathrm{P}} \Delta w\right)^{T}\left(r+\alpha^{\mathrm{aff}, \mathrm{D}} \Delta r\right)\right] .
$$

Finally, the value of $\sigma$ is chosen to be

$$
\sigma=\left(\frac{\mu^{\mathrm{aff}}}{\mu}\right)^{3} .
$$

The actual search direction is obtained by simply adding the affine-scaling direction to the centering-corrector direction; that is,

$$
(\Delta x, \Delta \pi, \Delta s, \Delta r, \Delta w)=\left(\Delta x^{\mathrm{aff}}, \Delta s^{\mathrm{aff}}, \Delta r^{\mathrm{aff}}, \Delta w^{\mathrm{aff}}\right)+\left(\Delta x^{\mathrm{cc}}, \Delta \pi^{\mathrm{cc}}, \Delta s^{\mathrm{cc}}, \Delta r^{\mathrm{cc}}, \Delta w^{\mathrm{cc}}\right) .
$$

The step taken by the algorithm is then a fraction of the maximum steps $\alpha^{\max , \mathrm{P}}, \alpha^{\max , \mathrm{D}}$ to the boundary in the primal and dual variables, respectively. Similarly to (14), we calculate

$$
\begin{aligned}
& \alpha^{\max , \mathrm{P}}=\inf \{\alpha \in[0,1] \mid(x, w)+\alpha(\Delta x, \Delta w) \geq 0\}, \\
& \alpha^{\max , \mathrm{D}}=\inf \{\alpha \in[0,1] \mid(s, r)+\alpha(\Delta s, \Delta r) \geq 0\},
\end{aligned}
$$

and set

$$
\alpha^{\mathrm{P}}=\gamma_{\mathrm{P}} * \alpha^{\max , \mathrm{P}}, \quad \alpha^{\mathrm{D}}=\gamma_{\mathrm{D}} * \alpha^{\max , \mathrm{D}},
$$

where $\gamma_{\mathrm{P}}$ and $\gamma_{\mathrm{D}}$ are two scaling factors obtained from Mehrotra's adaptive steplength heuristic $[7$, p. 588].

Having described all the ingredients, we can summarize the algorithm as follows:

Given $\left(x^{0}, \pi^{0}, s^{0}, r^{0}, w^{0}\right)$ with $\left(x^{0}, s^{0}, r^{0}, w^{0}\right)>0$

for $k=0,1,2, \ldots$

if termination test is satisfied

$$
\text { stop; }
$$

Set $(x, \pi, s, r, w)=\left(x^{k}, \pi^{k}, s^{k}, r^{k}, w^{k}\right)$ and calculate the affine-scaling direction

from (9) by setting the right-hand side as in (10), (11);

calculate $\alpha^{\text {aff,P }}, \alpha^{\text {aff,D }}, \mu^{\text {aff }}$ and $\sigma$ from (14), (15), and (16);

Calculate the centering-corrector step from (9) by setting the right-hand

side as in (12);

Calculate the search direction from (17);

Calculate $\alpha^{\mathrm{P}}, \alpha^{\mathrm{D}}$ from (18) and (19);

Calculate new iterate as 


$$
\begin{aligned}
\left(x^{k+1}, w^{k+1}\right) & =(x, w)+\alpha^{\mathrm{P}}(\Delta x, \Delta w) \\
\left(\pi^{k+1}, s^{k+1}, r^{k+1}\right) & =(\pi, s, r)+\alpha^{\mathrm{D}}(\Delta \pi, \Delta s, \Delta r)
\end{aligned}
$$

end (for).

Gondzio's [4] higher-order correction strategy is used to enhance the search direction at each iteration. In this approach, additional centering/correction directions are computed by solving (9) for different right-hand sides. Rather than attempting to correct the current point to the central path in a single step, Gondzio's strategy is more conservative, aiming only to bring the pairwise products $x_{i} s_{i}, i=1,2, \ldots, n$ and $r_{i} w_{i}, i \in \mathcal{U}$ that are much larger than the average $\mu$ more into line. The number of centering/correction directions depends on the ratio of time required to form and factor the coefficient matrix of the main linear system (see Section 4) to the time required to perform triangular substitutions with the factors. This ratio is machine dependent and therefore leads to different results on different architectures. We refer the interested reader to Gondzio's paper for details. Our implementation draws not only on this paper and also on Gondzio's code HOPDM (version 2.13), in which slightly different heuristics from those described in the paper are used.

Our code applies the scaling technique of Curtis and Reid [2] to the coefficient matrix $A$ before solving. This technique aims to minimize the deviation of the nonzero elements in the matrix from 1 , which it measures by the objective function

$$
\sum_{A_{i j} \neq 0} \log ^{2}\left|A_{i j}\right|
$$

It finds row and column scaling factors $\rho_{i}, i=1,2, \ldots, m$ and $\chi_{j}, j=1,2, \ldots, n$ such that the scaled version of $A$ (whose elements are $A_{i j} /\left(\rho_{i} \chi_{j}\right)$ ) minimizes this objective. Conjugate gradient turns out to be very effective when applied to the least squares problem of finding the $\rho_{i}$ and $\chi_{j}$ factors, and convergence to an approximate solution of adequate accuracy is usually achieved in three or four iterations.

Scaling generally improves the efficiency of the algorithm, but occasionally results in poorer performance. It can be disabled by the user, as we show in Section 6 .

The algorithm terminates in one of four states: optimal, infeasible, unknown, and suboptimal. Optimal termination occurs when the current iterate satisfies the following tests:

$$
\begin{aligned}
\frac{\left\|\left(r_{b}, r_{u}\right)\right\|}{1+\left\|\left(b^{T}, u^{T}\right)\right\|} & \leq \text { prifeastol, } \\
\frac{\left\|r_{c}\right\|}{1+\|c\|} & \leq \text { dualfeastol, } \\
\frac{\left|c^{T} x-\left(b^{T} \pi-\sum_{i \in \mathcal{U}} u_{i} r_{i}\right)\right|}{1+\left|c^{T} x\right|} & \leq \text { opttol, }
\end{aligned}
$$

where prifeastol, dualfeastol, and opttol are three tolerances whose default values are $10^{-8}$, $10^{-8}$, and $10^{-8}$, respectively.

For the remaining termination conditions, we make use of a merit function $\phi$ defined by

$$
\phi(\pi, x, s, w, r)=\frac{\left\|\left(r_{b}, r_{u}\right)\right\|}{\max (1,\|(b, u)\|)}+\frac{\left\|r_{c}\right\|}{\max (1,\|c\|)}+\frac{\left|c^{T} x-\left(b^{T} \pi-\sum_{i \in \mathcal{U}} u_{i} r_{i}\right)\right|}{\max (1,\|(b, u)\|,\|c\|)}
$$


Clearly, points $(\pi, x, s, w, r)$ at which $(x, s, w, r) \geq 0$ and $\phi=0$ are primal-dual solutions of (6), (7) and vice versa. When applied to feasible linear programs (for which a primal-dual solution is known to exist), $\phi$ typically decreases steadily to zero after perhaps oscillating during the first few iterations. We also maintain an array $\phi_{\min }$ whose $k$ th element is the smallest value of $\phi$ encountered up to iteration $k$; that is,

$$
\phi_{\min }[k]=\min _{\ell=0,1, \ldots, k} \phi\left(\pi_{\ell}, x_{\ell}, s_{\ell}, w_{\ell}, r_{\ell}\right) .
$$

Infeasible problems (that is, problems for which no primal-dual solutions exist) can be detected fairly reliably by a sharp increase in $\phi$. We terminate the algorithm at iteration $k$ with status infeasible if it fails the optimality test above but satisfies

$$
\phi\left(\pi_{k}, x_{k}, s_{k}, w_{k}, r_{k}\right) \geq \max \left(10^{-8}, 10^{5} \phi_{\min }[k]\right) .
$$

In other situations, the code is unable to resolve the question of feasibility. It exhibits slow convergence, or else the improvement in duality measure $\mu$ far outstrips the improvement in primal and dual infeasibility $\left(\left\|\left(r_{b}, r_{u}\right)\right\|\right.$ and $\left\|r_{c}\right\|$, respectively), causing $\mu$ to lose its relationship to the true gap between the primal and dual objective function values. In both these cases, we terminate the algorithm with status unknown. The slow convergence test is

$$
\phi_{\min }[k-30] \geq \frac{1}{2} \phi_{\min }[k] \quad \text { and } k \geq 30 .
$$

Blowup in infeasibility-to-duality ratio is flagged if we have

$$
\frac{\left\|\left(r_{b}^{k}, r_{u}^{k}\right)\right\|}{1+\left\|\left(b^{T}, u^{T}\right)\right\|}>\text { prifeastol or } \frac{\left\|r_{c}^{k}\right\|}{1+\|c\|}>\text { dualfeastol, }
$$

and, in addition,

$$
\frac{\max \left(\left\|\left(r_{b}^{k}, r_{u}^{k}\right)\right\|,\left\|r_{c}^{k}\right\|\right) / \mu_{k}}{\max \left(\left\|\left(r_{b}^{0}, r_{u}^{0}\right)\right\|,\left\|r_{c}^{0}\right\|\right) / \mu_{0}} \geq 10^{6}
$$

Finally, we terminate in suboptimal status if the algorithm exceeds its allotted maximum number of iterations (see iterationlimit in Section 6) without satisfying any of the conditions above.

\section{Linear Algebra}

The coefficient matrix in (9) is sparse and highly structured. With the exception of the $A$ and $A^{T}$ blocks, all blocks are either zero or diagonal. By performing simple block elimination on this system, we obtain the following alternative formulation of the step equations, known as the augmented system form:

$$
\left[\begin{array}{cc}
-D^{-2} & A^{T} \\
A & 0
\end{array}\right]\left[\begin{array}{c}
\Delta x \\
\Delta \pi
\end{array}\right]=\left[\begin{array}{c}
-r_{c}-W^{-1} r_{w r}+X^{-1} r_{x s}+W^{-1} R r_{u} \\
-r_{b}
\end{array}\right]
$$

where $D$ is the positive diagonal matrix defined by $D=\left(S^{-1} X+W^{-1} R\right)^{1 / 2}$. The remaining components $\Delta w, \Delta r$, and $\Delta s$ of the solution vector can be recovered as follows:

$$
\begin{aligned}
\Delta w & =-r_{u}-\Delta x \\
\Delta r & =-W^{-1}\left(R \Delta w+r_{w r}\right) \\
\Delta s & =-X^{-1}\left(S \Delta x+r_{x s}\right) .
\end{aligned}
$$


The system (21) can be reduced to an even more compact form as follows by eliminating $\Delta x$ to obtain

$$
A D^{2} A^{T} \Delta \pi=-r_{b}+A D^{2}\left(-r_{c}-W^{-1} r_{w r}+X^{-1} r_{x s}+W^{-1} R r_{u}\right) .
$$

The component $\Delta x$ can be recovered from

$$
\Delta x=D^{2}\left(A^{T} \Delta \pi-\left(-r_{c}-W^{-1} r_{w r}+X^{-1} r_{x s}+W^{-1} R r_{u}\right)\right),
$$

while the remaining step components can be obtained as before from (22).

PCx uses the formulation (23), which is often known as the normal equations form. A sparse Cholesky algorithm is used to factor the coefficient matrix $A D^{2} A^{T}$, and the solution $\Delta \pi$ is obtained by performing triangular substitutions with the Cholesky factor $L$. These factorizations and triangular substitutions dominate the computational cost of the algorithm. The factorization is carried out with the sparse Cholesky code of $\mathrm{Ng}$ and Peyton [8], modified slightly to handle the small pivot elements that frequently arise during later iterations of the interior-point method. This code produces a factorization of the form

$$
P\left(A D^{2} A^{T}\right) P^{T}=L L^{T},
$$

where $P$ is a permutation matrix (determined independently of the numerical values in $A D^{2} A^{T}$ during an ordering step) and $L$ is a lower triangular matrix.

$\mathrm{Ng}$ and Peyton's code uses a multiple minimum degree ordering strategy identical to the one in SPARSPAK. This strategy was introduced by Liu [5]. The scheme used for symbolic factorization is partly described by Liu [6] and Gilbert, $\mathrm{Ng}$, and Peyton [3]. The numerical factorization is performed by a left-looking block sparse Cholesky algorithm, as described by $\mathrm{Ng}$ and Peyton [8]. The code exploits hierarchical memory by splitting the supernodes into blocks that fit into available cache. (Cache size is passed to the code as a parameter.) Loop unrolling is used to make better use of registers.

The release of $\mathrm{Ng}$ and Peyton's code used here is version 0.4 of May 1995 .

Since the nonzero structure of the matrix that we factor is the same at each interior-point iteration, the ordering and symbolic factorization operations are carried out just once, during computation of the initial point. At each interior-point iteration, the numerical factorization is performed once. Two back-substitutions are performed with these computed factors: one for the affine-scaling step, and one for the corrector-centering step.

Our modification of the Ng-Peyton code for small pivots requires just a handful of additional lines of Fortran. A candidate pivot $M_{i i}^{(i-1)}$ is deemed to be "small" if

$$
M_{i i}^{(i-1)} \leq 10^{-30} \max _{j=1,2, \cdots, m} M_{j j}^{2}
$$

where $M^{(i-1)}$ is the remaining submatrix after $i-1$ steps of the Cholesky factorization and $M$ is the original symmetric positive semidefinite matrix. Each small pivot is replaced by the very large number $10^{128}$. This substitution causes the off-diagonal elements in the $i$ th column of the Cholesky factor $L$ to be extremely small (essentially zero) and causes the $i$ th component of the solution vector to be extremely small. Analysis of this technique has been performed by Wright [9].

A similar pivot modification strategy is used by the MATLAB-based code LIPSOL (see Zhang $[11],[12])$, which also uses $\mathrm{Ng}$ and Peyton's code as its computational engine. 
If the matrix $A$ contains dense columns, the product $A D^{2} A^{T}$ may be much denser than $A$ itself, causing the unadorned normal equations strategy to be inefficient. We modify this strategy by excluding the dense columns from the computation of $A D^{2} A^{T}$ and accounting for them instead by using the Sherman-Morrison-Woodbury inverse updating formula. At the start of the PCx algorithm, during computation of the initial point, we partition $A$ into "sparse" and "dense" column submatrices $A_{\mathrm{sp}}$ and $A_{\mathrm{den}}$, respectively. The diagonal weighting matrix $D$ can be partitioned accordingly into $D_{\mathrm{sp}}$ and $D_{\text {den }}$, so we can write

$$
A D^{2} A^{T}=A_{\mathrm{sp}} D_{\mathrm{sp}}^{2} A_{\mathrm{sp}}^{T}+A_{\mathrm{den}} D_{\mathrm{den}}^{2} A_{\mathrm{den}}^{T}=M+A_{\mathrm{den}} D_{\mathrm{den}}^{2} A_{\mathrm{den}}^{T}
$$

where we have defined $M$ in an obvious way. By applying the Sherman-Morrison-Woodbury formula to (27), we find that

$$
\begin{aligned}
& {\left[M+A_{\mathrm{den}} D_{\mathrm{den}}^{2} A_{\mathrm{den}}^{T}\right]^{-1}} \\
& \quad=M^{-1}-\left(M^{-1} A_{\mathrm{den}}\right)\left[D_{\mathrm{den}}^{-1}+A_{\mathrm{den}}^{T} M^{-1} A_{\mathrm{den}}\right]^{-1} A_{\mathrm{den}}^{T} M^{-1} .
\end{aligned}
$$

We apply the sparse Cholesky procedure to $M$ alone, to obtain

$$
P M P^{T}=L L^{T},
$$

(cf. (25)). The solution of a linear system with coefficient matrix $M$ and right-hand side $r$ can now be written as

$$
\begin{aligned}
& \left(A D^{2} A^{T}\right)^{-1} r \\
& =P^{T} L^{-T}\left\{I-L^{-1} P A_{\operatorname{den}}\left[D_{\text {den }}^{-2}+A_{\text {den }}^{T} P^{T} L^{-T} L^{-1} P A_{\text {den }}\right]^{-1} A_{\text {den }}^{T} P^{T} L^{-T}\right\} L^{-1} \operatorname{Pr} .
\end{aligned}
$$

Given $L$ and $P$, the major costs of applying this formula are the cost of computing $\left(L^{-1} P A_{\mathrm{den}}\right)$, the cost of a triangular substitution with $L$ and one with $L^{T}-$ a total cost of $n_{\text {den }}+2$ triangular substitutions, where $n_{\text {den }}$ is the number of columns in $A_{\text {den }}$. For additional systems with the same coefficient matrix but different right-hand sides, the marginal cost is just two triangular substitutions.

To determine which columns are to be classified as "dense," we sort in decreasing order an array whose components are the number of nonzeros in each column. We then look at the columns for which the proportion of nonzeros is at least $\tau$, where $\tau=1$ for $m<500, \tau=0.1$ for $500<m \leq 2000$, and $\tau=0.05$ for $m>2000$, and try to identify a gap in the sequence of nonzero counts. (In our experience, most problems that benefit from special handling of the dense columns exhibit such a gap.) Columns whose nonzero counts lie on the high side of the gap are classified as "dense."

Another feature of PCx version 1.0 is the use of a preconditioned conjugate gradient (PCG) algorithm to improve the accuracy of computed solutions for the linear system (23). Essentially, we use the computed Cholesky factorization (25) (or (29)) as the preconditioner and treat the computed solution as the first iteration of a PCG algorithm. The PCG algorithm is activated if the computed solution fails to reduce the residual by a factor primalfeastol or better (see Section 6), and if no small pivot modifications are required during the Cholesky factorization. If dense columns are detected in $A$, PCG terminates when the residual reduction factor primalfeastol is achieved, or after a maximum of $10 n_{\text {den }}$ PCG iterations, whichever comes first. If no dense columns are present in $A$, at most 10 PCG iterations are allowed. 


\section{The Presolver}

Linear programming models frequently contain redundant information, as well as other information and structure that allows some components of the solution to be determined without recourse to a sophisticated algorithm. The purpose of presolve or preprocessing routines is to detect and handle these features of the input, producing a (smaller) problem to be solved by the actual linear programming algorithm. Presolvers significantly enhance the efficiency and robustness of both simplex and interior-point codes.

The presolver in PCx works with the formulation (1) stored in the LPtype data structure. It makes use of techniques described by Andersen and Andersen [1], checking the data for the following features:

Infeasibility. Check that $u_{i} \geq 0$ for each upper bound $u_{i}, i \in \mathcal{U}$, and that a zero row of $A$ has a corresponding zero in the right-hand side vector $b$.

Empty Rows. If the matrix $A$ has a zero row and a corresponding zero in the $b$, it can be removed from the problem.

Duplicate Rows. When a row of $A$ (and the corresponding element of the right-hand side $b$ ) is simply a multiple of another row, we can delete it without affecting the primal solution.

Duplicate Columns. When a column of $A$ is a multiple of another column, and if the two variables $x_{i}$ and $x_{j}$ are "normal" (that is, $i, j \in \mathcal{N}$ in the formulation (2)), the two columns can be combined. The primal variable for the combined column is either normal or free, depending on whether the columns are positive or negative multiples of each other.

Empty Columns. The corresponding element $x_{i}$ can be fixed at either its lower or upper bound, depending on the sign of the cost vector coefficient $c_{i}$. If the required bound does not exist, the problem is declared to be primal unbounded.

Fixed Variables. If the variable has lower and upper bounds both zero, it can obviously be fixed at zero and removed from the problem.

Singleton Rows. If the $i$ th row of $A$ contains the single nonzero element $A_{i j}$, we clearly have $x_{j}=b_{i} / A_{i j}$, so this variable can be removed from the problem. The $i$ th row of $A$ (and hence the dual variable $\pi_{i}$ ) can also be removed.

Singleton Columns. When $A_{i j}$ is the only nonzero in column $j$ of $A$, and $x_{j}$ is a free variable, we can express $x_{j}$ in terms of the other variables represented in row $i$ of $A$ and eliminate it from the problem. Even if not free, $x_{j}$ can be eliminated if its bounds are weaker than those implied by the ranges of the other elements represented in the row $A_{i}$..

Forced Rows. Sometimes, the linear constraint represented by row $i$ of $A$ forces all its variables to either their upper or lower bounds. An example would be the constraint $10 x_{3}-4 x_{10}+$ $x_{12}=-4$ subject to the bounds

$$
x_{3} \in[0,+\infty), \quad x_{10} \in[0,1], \quad x_{12} \in[0,+\infty) .
$$

In this case, we must have $x_{3}=0, x_{10}=1$ and $x_{12}=0$, so these three variables (and the corresponding row of $A$ ) can be eliminated. 
The presolver makes multiple passes through the data, checking for each of the above features in turn. Problem reductions on one pass frequently uncover further reductions that are detected on subsequent passes. The presolver terminates when a complete pass is performed without detecting further opportunities for reduction. Each reduction operation is pushed onto a stack, which is subsequently popped after the solution of the reduced linear program is found. The effect of popping the stack is to express the solution in terms of the original, unreduced formulation.

Despite the complexity of the code, the presolver requires little CPU time in comparison with a single iteration of the interior-point solver.

Code for the presolver can be found in the file presolve.c. The data structures are defined in pre.h. This code can be used on a stand-alone basis, independently of the PCx solver, to presolve any linear program supplied in the LPtype format.

\section{Specifications File}

PCx allows many algorithmic parameters and options to be set by the user. These quantities are stored internally in a data structure of type Parameters.

If the user provides input to $\mathrm{PCx}$ via an MPS file (rather than invoking $\mathrm{PCx}($ ) directly via a subroutine call), the Parameters data structure is allocated automatically by the program and default values are assigned to all parameters. You can override the default values by defining a specifications file, which contains a number of keywords and numerical values.

$\mathrm{PCx}$ searches for the specifications file in a number of locations. If the name of the MPS input file is probname.mps, $\mathrm{PCx}$ looks for the following files, in order:

$$
\text { probname.spc, probname.specs, spc, specs, PCx.specs }
$$

If more than one of these files exist, PCx uses the first file in the list above and ignores the others.

The following is a list of keywords that can be used in the specifications file, together with their default settings. The file should contain one such keyword per line, together with its corresponding numerical value or option, if appropriate. The file is processed sequentially from top to bottom, so the effect of any line in the file can be undone by a later line. For keywords with a yes/no argument, omission of the argument will be taken to mean yes. (The default setting is not necessarily yes.) In the descriptions below, we assume that PCX is invoked with the command

PCx probname

boundname \{name Request the bound to be the specific column name in probname.mps. Default: the first BOUND in the MPS file is used.

cachesize $\{$ value $\}$ Input the size of the cache on the machine, in Kilobytes. Any value in the range $0-2048$ is acceptable. Specify 0 for Cray machines. This parameter is used by the Ng-Peyton sparse Cholesky code. Default: 16.

centerexp \{value Specify the exponent to be used for calculation of the centering parameter $\sigma$ in (16). Any real value in the range $1.0-4.0$ is allowable. Default: 3.0 .

dualfeastol $\{$ value $\}$ Specify a dual feasibility tolerance. Default: $10^{-8}$. 
history $\{$ yes $\} /\{$ no $\}$ Request that a history file be written (yes) or not written (no). If yes, the file probname. $\log$ is written to the working directory (see Section 8 ).

HOCorrections $\{$ yes $\} /\{$ no $\}$ Request that Gondzio's [4] higher-order corrections be used to enhance the search direction. Default: yes.

inputdirectory \{name Give the directory here if $\mathrm{PCx}$ is to search for the MPS input files in some directory other than the working directory, give the directory here. Remember to include a trailing "/". If PCx cannot file the file in the specified input directory, it will look in the working directory. Whether or not an inputdirectory is specified, the output and history files are written to the working directory.

iterationlimit \{value\} An upper limit on the number of iterations. Any positive integer is allowable. Default: 100.

$\max$ Maximize the objective.

MaxCorrections \{value\} If HoCorrections = yes, the parameter MaxCorrections is an upper limit on the number of Gondzio's higher-order corrections allowed at each iteration. If value $=0$, the maximum is determined automatically by $\mathrm{PCx}$ according to the relative cost of factorization and solve operations. If HoCorrections $=$ no, MaxCorrections is ignored. Default: 0 .

min Minimize the objective (default).

objectivename \{name\} Request the objective cost vector to be the specific row name in probname.mps.

Default: the first row of type " $N$ " in probname.mps is taken to be the objective.

opttol \{value\} Specify an optimality tolerance. Default: $10^{-8}$.

preprocess $\{$ yes $\} /\{$ no $\}$ Synonymous with presolve.

presolve $\{$ yes $\} /\{$ no $\}$ Request that presolving be performed (yes) or not performed (no) (see Section 5). Default: yes.

prifeastol \{value\} Specify a primal feasibility tolerance. Default: $10^{-8}$.

rangename \{name\} Request the range to be the specific column name in probname.mps. Default: the first range encountered in the MPS file is used.

refinement $\{$ yes $\} /\{$ no $\}$ Perform preconditioned conjugate gradient refinement of the computed solution to the linear system (23) if it has a relative residual larger than the parameter prifeastol (yes) or don't perform any iterative refinement (no) (see Section 4). Default: no.

rhsname \{name Request the right-hand side to be the specific column name in probname.mps. Default: the first RHS encountered in the MPS file is used.

scaling $\{$ yes $\} /\{$ no $\}$ If yes, row and column scaling is performed on the constraint matrix. Default: yes. 
solution $\{$ yes $\} /\{$ no $\}$ Request that a solution file be written (yes) or not written (no). If the solution file is written, it is named probname.out and is placed in the working directory (see Section 8). Default: yes.

stepfactor $\{$ value $\}$ Specify a value in the range $(0,1)$ that is used in Mehrotra's adaptive steplength heuristic from [7, p. 118]. This value is a lower bound for $\gamma^{\mathrm{P}}$ and $\gamma^{\mathrm{D}}$ in (19). Default: 0.9 .

unrollinglevel \{value\} Specify the level of loop unrolling. Allowable values are 1, 2, 4, and 8. (This parameter is used only in the Ng-Peyton sparse Cholesky code.) Default: 4.

If you call $\mathrm{PCx}()$ directly from your own code, you must fill out the Parameters data structure explicitly. This task is easier if you use the routine $*$ NewParameters ( ) to allocate the storage, since this routine assigns default values to all parameters. You can then make any desired alterations before passing the data structure to the $\mathrm{PCx}()$ routine.

\section{Obtaining and Installing PCx}

PCx contains material protectable under copyright laws of the United States. Permission is hereby granted to use, reproduce, prepare derivative works, and redistribute to others at no charge, provided that any changes are clearly documented and that the original $\mathrm{PCx}$ copyright notice, Government license and disclaimer are retained; however, any entity desiring permission to incorporate this software, or a work based on this software, into a product for sale must contact Paul Betten at the Industrial Technology Development Center, Argonne National Laboratory, Argonne, IL 60439 (phone: 630/252-4962,fax: 630/252-5230, email: betten@anl.gov). For further information, refer to the copyright notice on the software.

The source code and documentation for $\mathrm{PCx}$ can be obtained through the World Wide Web and anonymous ftp. The $\mathrm{PCx}$ home page is

$$
\text { http://www.mcs.anl.gov/home/otc/Library/PCx/ }
$$

This page lists the Unix systems on which PCx has been compiled and tested, and also contains the copyright statement. The PCx home page also links to the following three files:

PCx.tar.gz: A gzipped tar file containing the source code, a Makefile, and a README file containing installation instructions. It also contains a postscript version of this user guide.

PCx-user.ps: A postscript version of this user guide.

results.ps: The tables of computational results from Section 9 of this guide.

Executables for PCx for the SunOS, Solaris, IBM RS/6000 AIX, and SGI IRIX Unix environments can be built from source via the following procedure. Download the file PCx.tar.gz and place it in its own subdirectory (referred to henceforth as the "working directory"). From the working directory, unzip the file by typing

$$
\text { gunzip PCx.tar.gz }
$$

\footnotetext{
${ }^{1}$ gunzip can be downloaded from $\mathrm{ftp}: / /$ quest.jpl.nasa.gov/pub/for compilation on a variety of architectures.
} 
and then un-tar the resulting file by typing

$\operatorname{tar} x v f \mathrm{PCx}, \operatorname{tar}$

The subdirectories SRC/, DOC/, MAKEARCH/, Ng-Peyton/, mps/ will be created by the tar command. A sample specifications file named PCx.specs and a number of executable script files will also appear. To create the executable PCx in the working directory, type

build

Because of architectural and environmental differences, it is necessary to have a slightly different compilation procedure for each machine. The build script defines an environment variable PCx_ARCH and assigns it a value to indicate the architecture. build then invokes the make procedure, with architecture-dependent portions of the makefile being retrieved from the subdirectory MAKEARCH/. Since the variable PCX_ARCH must be defined for compiling, one should always use build instead of make to compile the program.

Executables are also available for the SunOS, Solaris, AIX, and IRIX systems. The PCx Web page also contains links to these files.

To test $\mathrm{PCx}$ it on one of the input files in the directory mps/, modify the sample specifications file PCx.specs if desired, then type

$\mathrm{PCx}$ afiro

or

$\mathrm{PCx} 25 \mathrm{fv} 47$

The program and documentation files can also be retrieved via anonymous ftp. Go to ftp.mcs.anl.gov and cd to pub/neos/PCx. The files mentioned above can be found at:

ftp://ftp.mcs.anl.gov/pub/neos/PCx/PCx.tar.gz

ftp://ftp.mCs.anl.gov/pub/neos/PCx/PCx-user.ps

ftp://ftp.mcs.anl.gov/pub/neos/PCx/results/results.ps

The executables can be found at the following URLs:

ftp://ftp.mcs.anl.gov/pub/neos/PCx/sun4/PCxexe.tar.gz (SunOS)

ftp://ftp.mcs.anl.gov/pub/neos/PCx/solaris/PCxexe.tar.gz (Solaris)

ftp://ftp.mcs.anl.gov/pub/neos/PCx/rs6000/PCxexe.tar.gz (RS/6000 AIX)

ftp://ftp.mcs.anl.gov/pub/neos/PCx/irix/PCxexe.tar.gz (SGI IRIX)

ftp://ftp.mcs.anl.gov/pub/neos/PCx/hp/PCxexe.tar.gz (Hewlett-Packard HPUX)

ftp://ftp.mcs.anl.gov/pub/neos/PCx/linux/PCxexe.tar.gz (PC Linux)

These executables can be gunzip-ed and tar-ed as described above to produce an executable named PCx.

The transfer mode should be set to binary by using the bin command in ftp before attempting to transfer PCx.tar.gz or any of the executable files. 


\section{Invoking $\mathbf{P C x}$}

By downloading and installing PCx on one's system (see Section 7), the user will have an executable PCx, a Makefile and a build script in the current working directory, together with a number of subdirectories containing source files for $\mathrm{PCx}$, object files, documentation, and source files and a library for the Ng-Peyton sparse Cholesky code.

To solve a linear program contained in the MPS file probname.mps, one should go to the working directory (that is, the directory in which the executable $\mathrm{PCx}$ resides) and type

PCx probname

The file probname.mps can reside either in the working directory or in an "input directory" defined in the specifications file (see Section 6). PCx first searches the input directory (if specified) for the given file. It searches for the file name both with and without the .mps extension. If it does not find the file in the input directory, it searches the working directory.

PCx optionally produces two output files named probname.out and probname.1og, according to the options supplied by the user in the specifications file (see Section 6 ). These files are written in the working directory. They contain, respectively, the primal-dual point returned by the algorithm (provided the termination status is not infeasible), and a summary of iteration history, timings, preprocessor results, and sparsity statistics for the Cholesky factorization. Output is also written to standard output during execution of PCx. Essentially, the on-screen output consists of the information written to the file probname.log, together with error messages and warnings.

When PCx is executed as a standalone system and a runtime error is detected, the code returns a nonzero integer to the operating system. The return status indicates the type of error, as follows:

1: invocation error for PCx;

2: memory allocation error (usually, insufficient storage available);

3: error in the MPS input file;

4: error in the specifications file;

5: error detected during presolve; or

6: error encountered during matrix factorization, conjugate gradient iteration, sparse matrix multiplication, or dense column linear algebra.

The subroutine $\mathrm{PCx}()$ can also be invoked directly from user-written code. In this case, the user should fill out data structures that define the linear program and the algorithmic parameters. See the source code and the comments therein for details of this mode of use.

\section{Computational Results}

We have executed PCx version 1.0 successfully in a variety of Unix environments, including IBM RS6000/370 workstation running AIX, with 128 MB main memory and 350 MB swap space, running AIX; 
Sun SPARCstation-5 running SunOS4.3, with $32 \mathrm{MB}$ main memory;

Sun UltraSparc 2 running Solaris 2.x, with $200 \mathrm{MHz}$ processor, $1 \mathrm{MB}$ cache and $256 \mathrm{MB}$ of main memory;

SGI workstation running IRIX 5.3, with $250 \mathrm{MHz}$ processor, $2 \mathrm{MB}$ L2-cache, $64 \mathrm{MB}$ main memory.

HP9000-735 workstation running HPUX-9.05, with $128 \mathrm{MB}$ main memory and $125 \mathrm{MHz}$ PA7150 chip.

Pentium Pro PC running PC Linux, with $48 \mathrm{MB}$ main memory.

We report results from the SGI machine. On this machine, the code was compiled with the default Fortran and $\mathrm{C}$ compilers ( $\mathrm{x} I \mathrm{f}$ and $\mathrm{cc}$, respectively), using the -0 optimization flag in both cases.

We solved a large set of test problems, both feasible and infeasible, taken for the most part from the familiar netlib set. Results obtained with the default parameter settings are shown in Tables 1-3. Each row in the tables contains the dimensions of the problem before and after presolving, measures of infeasibility and complementarity, the primal objective of the point returned by PCx, the maximum number of additional centering/corrector steps allowed at each iteration (over and above the single centering/corrector step of Mehrotra's algorithm), the number of iterations, and the CPU time. The tabulated infeasibility measure is a relative measure defined as

$$
\max \left(\frac{\left\|\left(r_{b}, r_{u}\right)\right\|}{1+\left\|\left(b^{T}, u^{T}\right)\right\|}, \frac{\left\|r_{c}\right\|}{1+\|c\|}\right)
$$

where $r_{b}, r_{u}$ and $r_{c}$ are the residuals at the final point. The tabulated complementarity measure is defined as

$$
\frac{x^{T} s+(u-x)^{T} r}{1+\left|c^{T} x\right|} .
$$

Results for the feasible problems are shown in Table 1 . In most cases, PCx correctly identified the problem as feasible and returned an optimal solution. In four cases, the code terminated with status unknown, though in three of these cases the point returned by the code is quite close to optimality. No problems were incorrectly flagged as infeasible.

Results for the infeasible problems appear in Tables 2. In two cases, $\mathrm{PCx}$ terminates with status unknown; the correct status infeasible is reported for all other problems. In two other cases, infeasibility was detected by the preprocessor, so the interior-point solver did not need to be called at all.

The NEMS problems are instances of models in the National Energy Modeling System (NEMS) of the Energy Information Administration of the United States Department of Energy [13]. These problems are taken from NEMS modules which are used to model electricity capacity planning, petroleum marketing, and coal marketing. PCx solved these problems efficiently, as shown in Tables 3.

The improvements obtained by using higher-order corrections are not too dramatic. Part of the reason is that the factorization routine is more efficient relative to the solution routine than is the case in, for example, HOPDM (see Gondzio [4]). It follows that there is less to be gained by economizing on matrix factorizations. Significant improvements can however be observed on several problems, including df1001, pds-10, NEMSemm1, and NEMSwrld. 
Computational results for the NETLIB test set and the NEMS problems on SGI workstation (250 $\mathrm{MHz}$ processor, L2-cache 2MB, Main memory 64MB, running IRIX 5.3)

Legend: ${ }^{*}=$ terminated with unknown status,

$\dagger=$ infeasibility detected during preprocessing,

max $=$ maximation problems

Table 1: NETLiB: Feasible NeTLiB Problems

\begin{tabular}{|c|c|c|c|c|c|c|c|c|c|c|}
\hline \multirow[b]{2}{*}{ Name } & \multicolumn{2}{|c|}{$\begin{array}{c}\text { Before } \\
\text { Preprocessing }\end{array}$} & \multicolumn{2}{|c|}{$\begin{array}{c}\text { After } \\
\text { Preprocessing }\end{array}$} & \multirow{2}{*}{$\begin{array}{c}\text { Relative } \\
\text { Infeas }\end{array}$} & \multirow{2}{*}{$\begin{array}{c}\text { Relative } \\
\text { Compl }\end{array}$} & \multirow{2}{*}{$\begin{array}{c}\text { Primal } \\
\text { Objective }\end{array}$} & \multirow{2}{*}{$\begin{array}{l}\text { Max } \\
\text { Add. } \\
\text { Corr. }\end{array}$} & \multirow[b]{2}{*}{ Iters } & \multirow{2}{*}{$\begin{array}{l}\text { CPU } \\
\text { Time } \\
\text { [secs] }\end{array}$} \\
\hline & Rows & Cols & Rows & Cols & & & & & & \\
\hline $25 \mathrm{fv} 47$ & 821 & 1876 & 788 & 1843 & $1.8 \mathrm{e}-11$ & $1.3 \mathrm{e}-07$ & $5.501846 e+03$ & 1 & 22 & 3.98 \\
\hline $80 \mathrm{bau} 3 \mathrm{~b}$ & 2262 & 12061 & 2140 & 11066 & $1.1 \mathrm{e}-11$ & $7.6 e-07$ & $9.872244 e+05$ & 0 & 37 & 13.07 \\
\hline adlittle & 56 & 138 & 55 & 137 & $8.2 \mathrm{e}-14$ & $3.4 \mathrm{e}-11$ & $2.254950 e+05$ & 0 & 12 & 0.05 \\
\hline afiro & 27 & 51 & 27 & 51 & $1.9 e-11$ & $7.2 \mathrm{e}-15$ & $-4.647531 \mathrm{e}+02$ & 0 & 8 & 0.01 \\
\hline agg & 488 & 615 & 390 & 477 & $2.5 e-09$ & $2.7 \mathrm{e}-12$ & $-3.599177 e+07$ & 0 & 19 & 0.87 \\
\hline agg2 & 516 & 758 & 514 & 750 & $3.4 e-11$ & $4.8 \mathrm{e}-10$ & $-2.023925 e+07$ & 1 & 20 & 1.97 \\
\hline agg3 & 516 & 758 & 514 & 750 & $4.5 e-11$ & $3.1 \mathrm{e}-11$ & $1.031212 \mathrm{e}+07$ & 1 & 19 & 1.99 \\
\hline bandm & 305 & 472 & 240 & 395 & $6.1 \mathrm{e}-13$ & $3.5 \mathrm{e}-12$ & $-1.586280 e+02$ & 0 & 17 & 0.38 \\
\hline beaconfd & 173 & 295 & 86 & 171 & $3.7 e-12$ & $2.4 \mathrm{e}-13$ & $3.359249 e+04$ & 1 & 10 & 0.16 \\
\hline blend & 74 & 114 & 71 & 111 & $4.6 \mathrm{e}-13$ & $5.7 \mathrm{e}-15$ & $-3.081215 \mathrm{e}+01$ & 0 & 10 & 0.06 \\
\hline bnl1 & 643 & 1586 & 610 & 1491 & $1.2 \mathrm{e}-11$ & $3.2 \mathrm{e}-08$ & $1.977630 e+03$ & 0 & 39 & 2.45 \\
\hline bnl2 & 2324 & 4486 & 1964 & 4008 & $4.4 e-09$ & $8.2 \mathrm{e}-08$ & $1.811237 \mathrm{e}+03$ & 1 & 31 & 17.07 \\
\hline boeing1 & 351 & 726 & 331 & 697 & $2.9 e-11$ & $1.8 \mathrm{e}-11$ & $-3.352136 e+02$ & 0 & 20 & 0.75 \\
\hline boeing2 & 166 & 305 & 126 & 265 & $2.1 \mathrm{e}-08$ & $1.1 \mathrm{e}-09$ & $-3.150187 e+02$ & 0 & 14 & 0.17 \\
\hline bore3d & 233 & 334 & 81 & 138 & $4.2 e-14$ & $4.8 \mathrm{e}-13$ & $1.373080 e+03$ & 0 & 16 & 0.11 \\
\hline brandy & 220 & 303 & 133 & 238 & $1.0 \mathrm{e}-05$ & $6.1 \mathrm{e}-15$ & $1.518510 \mathrm{e}+03$ & 1 & 16 & 0.37 \\
\hline capri & 271 & 482 & 241 & 436 & $9.6 \mathrm{e}-11$ & $1.4 \mathrm{e}-12$ & $2.690013 \mathrm{e}+03$ & 0 & 19 & 0.40 \\
\hline cycle & 1903 & 3371 & 1420 & 2773 & $5.7 \mathrm{e}-09$ & $4.9 \mathrm{e}-11$ & $-5.226393 e+00$ & 0 & 23 & 6.06 \\
\hline czprob & 929 & 3562 & 671 & 2779 & $3.7 \mathrm{e}-10$ & $1.1 \mathrm{e}-07$ & $2.185197 e+06$ & 0 & 26 & 1.61 \\
\hline $\mathrm{d} 2 \mathrm{q} 06 \mathrm{c}$ & 2171 & 5831 & 2132 & 5728 & $4.7 e-08$ & $2.8 \mathrm{e}-07$ & $1.227842 \mathrm{e}+05$ & 1 & 24 & 25.33 \\
\hline d6cube & 415 & 6184 & 403 & 5443 & $2.4 \mathrm{e}-09$ & $4.5 e-09$ & $3.154917 \mathrm{e}+02$ & 1 & 16 & 10.07 \\
\hline degen 2 & 444 & 757 & 444 & 757 & $7.4 \mathrm{e}-14$ & $8.4 e-13$ & $-1.435178 \mathrm{e}+03$ & 1 & 11 & 1.19 \\
\hline degen 3 & 1503 & 2604 & 1503 & 2604 & $5.6 \mathrm{e}-10$ & $8.0 \mathrm{e}-09$ & $-9.872940 e+02$ & 1 & 16 & 22.79 \\
\hline dfl001 & 6071 & 12230 & 5984 & 12143 & $1.5 \mathrm{e}-11$ & $2.7 \mathrm{e}-08$ & $1.126640 \mathrm{e}+07$ & 3 & 46 & 2857.07 \\
\hline e226 & 223 & 472 & 198 & 429 & $1.6 \mathrm{e}-12$ & $9.0 \mathrm{e}-10$ & $-2.586493 e+01$ & 0 & 18 & 0.44 \\
\hline etamacro & 400 & 816 & 334 & 669 & $1.4 e-14$ & $3.1 \mathrm{e}-08$ & $-7.557152 \mathrm{e}+02$ & 0 & 25 & 0.98 \\
\hline ffff 800 & 524 & 1028 & 322 & 826 & $5.8 e-10$ & $7.4 \mathrm{e}-08$ & $5.556796 \mathrm{e}+05$ & 1 & 25 & 1.91 \\
\hline finnis & 497 & 1064 & 438 & 935 & $4.2 \mathrm{e}-12$ & $1.1 \mathrm{e}-08$ & $1.727911 \mathrm{e}+05$ & 0 & 25 & 0.81 \\
\hline fit1d & 24 & 1049 & 24 & 1049 & $9.0 \mathrm{e}-14$ & $1.7 \mathrm{e}-07$ & $-9.146377 e+03$ & 1 & 17 & 1.66 \\
\hline fit1p & 627 & 1677 & 627 & 1677 & $3.3 e-09$ & $4.6 e-08$ & $9.146378 \mathrm{e}+03$ & 0 & 17 & 2.00 \\
\hline fit2d & 25 & 10524 & 25 & 10524 & $4.7 \mathrm{e}-14$ & $2.4 \mathrm{e}-08$ & $-6.846429 e+04$ & 1 & 23 & 25.10 \\
\hline fit $2 p$ & 3000 & 13525 & 3000 & 13525 & $4.4 \mathrm{e}-08$ & $1.8 \mathrm{e}-06$ & $6.846441 e+04$ & 0 & 19 & 16.09 \\
\hline forplan & 161 & 492 & 121 & 447 & $3.8 e-08$ & $3.9 \mathrm{e}-10$ & $-6.642190 e+02$ & 1 & 20 & 0.82 \\
\hline ganges & 1309 & 1706 & 1113 & 1510 & $4.4 \mathrm{e}-07$ & $9.7 e-09$ & $-1.095857 e+05$ & 0 & 17 & 2.25 \\
\hline gfrd-pnc & 616 & 1160 & 590 & 1134 & $9.7 e-15$ & $2.2 \mathrm{e}-11$ & $6.902236 \mathrm{e}+06$ & 0 & 18 & 0.55 \\
\hline greenbea & 2392 & 5598 & 1933 & 4164 & $1.5 \mathrm{e}-05$ & $1.2 \mathrm{e}-08$ & $-7.255525 e+07$ & 0 & 48 & 13.97 \\
\hline greenbeb * & 2392 & 5598 & 1932 & 4154 & $1.3 e-05$ & $3.4 \mathrm{e}-09$ & $-4.302260 \mathrm{e}+06$ & 0 & 39 & 11.33 \\
\hline grow15 & 300 & 645 & 300 & 645 & $3.3 e-07$ & $8.4 e-15$ & $-1.068709 e+08$ & 1 & 21 & 1.32 \\
\hline grow 22 & 440 & 946 & 440 & 946 & $4.1 e-05$ & $2.1 \mathrm{e}-10$ & $-1.608343 e+08$ & 1 & 22 & 2.27 \\
\hline grow 7 & 140 & 301 & 140 & 301 & $4.6 \mathrm{e}-09$ & $1.6 \mathrm{e}-09$ & $-4.778781 e+07$ & 1 & 17 & 0.50 \\
\hline israel & 174 & 316 & 174 & 316 & $1.4 \mathrm{e}-12$ & $1.3 \mathrm{e}-08$ & $-8.966448 \mathrm{e}+05$ & 1 & 19 & 1.30 \\
\hline kb2 & 43 & 68 & 43 & 68 & $3.0 e-10$ & $2.0 \mathrm{e}-16$ & $-1.749900 \mathrm{e}+03$ & 0 & 13 & 0.04 \\
\hline $\operatorname{lotfi}$ & 153 & 366 & 133 & 346 & $7.4 \mathrm{e}-10$ & 1.De-15 & $-2.526471 e+01$ & 0 & 15 & 0.15 \\
\hline maros & 846 & 1966 & 655 & 1437 & $2.8 \mathrm{e}-08$ & $7.1 e-11$ & $-5.806374 e+04$ & 0 & 20 & 1.54 \\
\hline maros-r 7 & 3136 & 9408 & 2152 & 7440 & $7.6 e-11$ & $2.2 \mathrm{e}-09$ & $1.497185 e+06$ & 1 & 15 & 89.20 \\
\hline $\bmod 2 *$ & 34774 & 66409 & 28760 & 56347 & $1.7 e-05$ & $4.5 e-05$ & $4.656998 \mathrm{e}+07$ & 0 & 78 & 554.80 \\
\hline modszk1 & 687 & 1620 & 665 & 1599 & $7.4 \mathrm{e}-09$ & $1.6 e-12$ & $3.206196 \mathrm{e}+02$ & 0 & 22 & 1.18 \\
\hline nesm & 662 & 3105 & 654 & 2922 & $3.4 \mathrm{e}-12$ & $1.4 \mathrm{e}-07$ & $1.407604 \mathrm{e}+07$ & 0 & 27 & 4.20 \\
\hline NL & 7039 & 15325 & 6665 & 14680 & $1.6 \mathrm{e}-12$ & $9.9 e-07$ & $1.229265 \mathrm{e}+06$ & 1 & 35 & 84.69 \\
\hline pds-10 & 16558 & 49932 & 15648 & 48780 & $2.6 \mathrm{e}-10$ & $3.5 e-06$ & $2.672717 \mathrm{e}+10$ & 3 & 35 & 1651.59 \\
\hline
\end{tabular}




\begin{tabular}{|c|c|c|c|c|c|c|c|c|c|c|}
\hline \multirow[b]{2}{*}{ Name } & \multicolumn{2}{|c|}{$\begin{array}{c}\text { Before } \\
\text { Preprocessing }\end{array}$} & \multicolumn{2}{|c|}{$\begin{array}{c}\text { After } \\
\text { Preprocessing }\end{array}$} & \multirow{2}{*}{$\begin{array}{l}\text { Relative } \\
\text { Infeas }\end{array}$} & \multirow{2}{*}{$\begin{array}{c}\text { Relative } \\
\text { Compl }\end{array}$} & \multirow{2}{*}{$\begin{array}{c}\text { Primal } \\
\text { Objective }\end{array}$} & \multirow{2}{*}{$\begin{array}{l}\text { Max } \\
\text { Add. } \\
\text { Corr. }\end{array}$} & \multirow[b]{2}{*}{ Iters } & \multirow{2}{*}{$\begin{array}{l}\text { CPU } \\
\text { Time } \\
\text { [secs] }\end{array}$} \\
\hline & Rows & Cols & Rows & Cols & & & & & & \\
\hline perold & 625 & 1506 & 593 & 1374 & $7.2 \mathrm{e}-07$ & $2.8 \mathrm{e}-11$ & $-9.380755 e+03$ & 0 & 34 & 3.35 \\
\hline pilot & 1441 & 4860 & 1368 & 4543 & $2.4 e-07$ & $4.5 e-07$ & $-5.574897 e+02$ & 1 & 32 & 72.18 \\
\hline pilot.ja & 940 & 2267 & 810 & 1804 & $2.5 \mathrm{e}-0.5$ & $3.0 \mathrm{e}-08$ & $-6.113136 e+03$ & 1 & 29 & 9.42 \\
\hline pilot.we & 722 & 2928 & 701 & 2814 & $2.4 \mathrm{e}-11$ & $1.2 \mathrm{e}-07$ & $-2.720107 e+06$ & 0 & 46 & 4.27 \\
\hline pilot4 & 410 & 1123 & 396 & 1022 & $4.8 \mathrm{e}-05$ & $5.1 e-09$ & $-2.581139 e+03$ & 1 & 46 & 4.74 \\
\hline pilot87 & 2030 & 6680 & 1971 & 6373 & $1.3 e-06$ & $6.7 e-07$ & $3.017105 e+02$ & 2 & 30 & 216.32 \\
\hline pilotnov & 975 & 2446 & 848 & 2117 & $4.0 \mathrm{e}-06$ & $1.3 e-11$ & $-4.497276 e+03$ & 1 & 16 & 4.98 \\
\hline radex & 16 & 26 & 15 & 25 & $3.7 e-13$ & $6.2 \mathrm{e}-14$ & $3.584229 \mathrm{e}+05$ & 0 & 8 & 0.01 \\
\hline recipe & 91 & 204 & 64 & 123 & $1.9 \mathrm{e}-10$ & $3.9 e-16$ & $-2.666160 \mathrm{e}+02$ & 0 & 9 & 0.05 \\
\hline $\operatorname{sc105}$ & 105 & 163 & 104 & 162 & $8.7 e-09$ & $1.5 \mathrm{e}-16$ & $-5.220206 e+01$ & 0 & 10 & 0.04 \\
\hline $\operatorname{sc} 205$ & 205 & 317 & 203 & 315 & $1.1 \mathrm{e}-11$ & $1.1 \mathrm{e}-13$ & $-5.220206 e+01$ & 0 & 11 & 0.10 \\
\hline sc50a & 50 & 78 & 49 & 77 & $2.4 e-11$ & $9.5 e-16$ & $-6.457508 e+01$ & 0 & 8 & 0.02 \\
\hline sc50b & 50 & 78 & 48 & 76 & $2.8 \mathrm{e}-09$ & $7.4 \mathrm{e}-11$ & $-7.000000 e+01$ & 0 & 6 & 0.02 \\
\hline scagr25 & 471 & 671 & 469 & 669 & $2.9 \mathrm{e}-12$ & $2.2 \mathrm{e}-13$ & $-1.475343 \mathrm{e}+07$ & 0 & 18 & 0.39 \\
\hline scagr 7 & 129 & 185 & 127 & 183 & $1.8 \mathrm{e}-13$ & $7.9 \mathrm{e}-09$ & $-2.331390 \mathrm{e}+06$ & 0 & 14 & 0.08 \\
\hline scfxm1 & 330 & 600 & 305 & 568 & $1.2 \mathrm{e}-06$ & $1.6 \mathrm{e}-09$ & $1.841676 \mathrm{e}+04$ & 0 & 17 & 0.45 \\
\hline $\operatorname{scfxm} 2$ & 660 & 1200 & 610 & 1136 & $2.2 \mathrm{e}-08$ & $5.3 e-13$ & $3.666026 e+04$ & 0 & 20 & 1.13 \\
\hline $\operatorname{scfxm} 3$ & 990 & 1800 & 915 & 1704 & $5.1 e-08$ & $3.6 e-12$ & $5.490125 e+04$ & 0 & 20 & 1.65 \\
\hline scorpion & 388 & 466 & 340 & 412 & $5.0 \mathrm{e}-14$ & $6.2 \mathrm{e}-16$ & $1.878125 \mathrm{e}+03$ & 0 & 12 & 0.20 \\
\hline scrs8 & 490 & 1275 & 421 & 1199 & $6.9 e-13$ & $1.1 \mathrm{e}-08$ & $9.042970 \mathrm{e}+02$ & 0 & 22 & 1.42 \\
\hline $\operatorname{scsd} 1$ & 77 & 760 & 77 & 760 & $7.3 e-15$ & $2.1 \mathrm{e}-15$ & $8.666667 \mathrm{e}+00$ & 0 & 9 & 0.18 \\
\hline $\operatorname{scsd} 6$ & 147 & 1350 & 147 & 1350 & $1.1 \mathrm{e}-14$ & $2.8 \mathrm{e}-09$ & $5.050000 e+01$ & 0 & 12 & 0.42 \\
\hline $\operatorname{scsd} 8$ & 397 & 2750 & 397 & 2750 & $6.4 \mathrm{e}-15$ & $9.2 \mathrm{e}-08$ & $9.050001 e+02$ & 0 & 11 & 0.75 \\
\hline sctap1 & 300 & 660 & 284 & 644 & $1.0 \mathrm{e}-13$ & $9.7 e-14$ & $1.412250 \mathrm{e}+03$ & 0 & 16 & 0.29 \\
\hline sctap2 & 1090 & 2500 & 1033 & 2443 & $2.3 \mathrm{e}-15$ & $4.0 \mathrm{e}-15$ & $1.724807 \mathrm{e}+03$ & 0 & 14 & 1.21 \\
\hline sctap 3 & 1480 & 3340 & 1408 & 3268 & $8.4 \mathrm{e}-16$ & $1.3 e-13$ & $1.424000 \mathrm{e}+03$ & 0 & 15 & 1.65 \\
\hline seba & 515 & 1036 & 448 & 901 & $4.7 \mathrm{e}-13$ & $6.8 \mathrm{e}-09$ & $1.571160 \mathrm{e}+04$ & 2 & 12 & 6.97 \\
\hline share1b & 117 & 253 & 112 & 248 & $2.6 \mathrm{e}-08$ & $3.4 e-10$ & $-7.658932 \mathrm{e}+04$ & 0 & 19 & 0.18 \\
\hline share $2 b$ & 96 & 162 & 96 & 162 & $2.1 \mathrm{e}-11$ & $1.6 \mathrm{e}-14$ & $-4.157322 e+02$ & 0 & 17 & 0.12 \\
\hline shell & 536 & 1777 & 487 & 1451 & $3.5 \mathrm{e}-13$ & $1.6 \mathrm{e}-11$ & $1.208825 \mathrm{e}+09$ & 0 & 21 & 0.72 \\
\hline ship04l & 402 & 2166 & 292 & 1905 & $5.8 \mathrm{e}-14$ & $2.8 \mathrm{e}-13$ & $1.793325 \mathrm{e}+06$ & 0 & 13 & 0.58 \\
\hline ship04s & 402 & 1506 & 216 & 1281 & $1.0 \mathrm{e}-11$ & $7.0 \mathrm{e}-09$ & $1.798715 \mathrm{e}+06$ & 0 & 13 & 0.38 \\
\hline ship081 & 778 & 4363 & 470 & 3121 & $5.7 e-14$ & $8.9 e-11$ & $1.909055 \mathrm{e}+06$ & $\mathbf{0}$ & 16 & 1.19 \\
\hline ship08s & 778 & 2467 & 276 & 1604 & $3.1 \mathrm{e}-13$ & $3.0 \mathrm{e}-11$ & $1.920098 \mathrm{e}+06$ & $\mathbf{0}$ & 12 & 0.48 \\
\hline ship12l & 1151 & 5533 & 610 & 4171 & $4.3 e-14$ & $2.0 \mathrm{e}-07$ & $1.470188 \mathrm{e}+06$ & 0 & 16 & 1.50 \\
\hline ship12s & 1151 & 2869 & 340 & 1943 & $5.0 \mathrm{e}-14$ & $3.6 \mathrm{e}-13$ & $1.489236 \mathrm{e}+06$ & $\mathbf{0}$ & 13 & 0.60 \\
\hline sierra & 1227 & 2735 & 1212 & 2705 & $4.8 \mathrm{e}-16$ & $8.0 e-10$ & $1.539436 \mathrm{e}+07$ & $\mathbf{0}$ & 21 & 2.05 \\
\hline stair & 356 & 614 & 356 & 532 & $1.3 e-09$ & $2.6 \mathrm{e}-08$ & $-2.512670 e+02$ & 1 & 13 & 1.02 \\
\hline standata & 359 & 1274 & 314 & 796 & $6.7 \mathrm{e}-15$ & $5.4 \mathrm{e}-09$ & $1.257700 \mathrm{e}+03$ & 0 & 13 & 0.27 \\
\hline standgub & 361 & 1383 & 314 & 796 & $6.7 e-15$ & $5.4 \mathrm{e}-09$ & $1.257700 \mathrm{e}+03$ & 0 & 13 & 0.27 \\
\hline standmps & 467 & 1274 & 422 & 1192 & $2.9 \mathrm{e}-14$ & $3.2 \mathrm{e}-15$ & $1.406017 \mathrm{e}+03$ & 0 & 26 & 0.79 \\
\hline stocfor1 & 117 & 165 & 102 & 150 & $4.5 e-13$ & $3.6 e-11$ & $-4.113198 \mathrm{e}+04$ & $\mathbf{0}$ & 12 & 0.06 \\
\hline stocfor 2 & 2157 & 3045 & 1980 & 2868 & $8.4 \mathrm{e}-11$ & $1.0 e-07$ & $-3.902441 \mathrm{e}+04$ & 0 & 20 & 2.37 \\
\hline stocfor3 & 16675 & 23541 & 15362 & 22228 & $2.3 e-09$ & $6.1 \mathrm{e}-08$ & $-3.997678 \mathrm{e}+04$ & 0 & 31 & 35.66 \\
\hline truss & 1000 & 8806 & 1000 & 8806 & $1.5 e-13$ & $1.8 \mathrm{e}-09$ & $4.588158 \mathrm{e}+05$ & 0 & 20 & 6.61 \\
\hline tuff & 333 & 628 & 257 & 567 & $3.0 \mathrm{e}-09$ & $2.9 e-13$ & $2.921478 \mathrm{e}-01$ & 0 & 20 & 0.82 \\
\hline vtp.base & 198 & 346 & 72 & 111 & $1.5 \mathrm{e}-08$ & $2.6 \mathrm{e}-09$ & $1.298315 e+0.5$ & 0 & 11 & 0.05 \\
\hline wood1p & 244 & 2595 & 171 & 1718 & $3.4 \mathrm{e}-11$ & $2.6 \mathrm{e}-11$ & $1.442902 \mathrm{e}+00$ & 2 & 20 & 12.31 \\
\hline woodw & 1098 & 8418 & 708 & 5364 & $6.2 \mathrm{e}-11$ & $1.2 \mathrm{e}-10$ & $1.304476 \mathrm{e}+00$ & 0 & 31 & 6.13 \\
\hline world * & 34506 & 67147 & 28652 & 58027 & $8.6 \mathrm{e}-03$ & $1.1 \mathrm{e}-04$ & $6.986991 e+07$ & 0 & 67 & 514.91 \\
\hline
\end{tabular}


Table 2: NethiB: Infeasible NetLiB Problems

\begin{tabular}{|c|c|c|c|c|c|c|c|c|c|c|}
\hline \multirow[b]{2}{*}{ Name } & \multicolumn{2}{|c|}{$\begin{array}{c}\text { Before } \\
\text { Preprocessing }\end{array}$} & \multicolumn{2}{|c|}{$\begin{array}{c}\text { After } \\
\text { Preprocessing }\end{array}$} & \multirow{2}{*}{$\begin{array}{l}\text { Relative } \\
\text { Infeas }\end{array}$} & \multirow{2}{*}{$\begin{array}{c}\text { Relative } \\
\text { Compl }\end{array}$} & \multirow{2}{*}{$\begin{array}{c}\text { Primal } \\
\text { Objective }\end{array}$} & \multirow{2}{*}{$\begin{array}{l}\text { Max } \\
\text { Add. } \\
\text { Corr. }\end{array}$} & \multirow[b]{2}{*}{ Iters } & \multirow{2}{*}{$\begin{array}{l}\text { CPU } \\
\text { Time } \\
\text { [secs] }\end{array}$} \\
\hline & Rows & Cols & Rows & Cols & & & & & & \\
\hline bgdbg1 & 348 & 629 & 249 & 509 & $1.8 e+02$ & $4.0 \mathrm{e}+00$ & $4.155802 \mathrm{e}+01$ & 0 & 6 & 0.12 \\
\hline bgetam & 400 & 816 & 334 & 669 & $8.4 e+01$ & $1.2 \mathrm{e}+01$ & $-3.571285 e+04$ & 0 & 7 & 0.35 \\
\hline bgindy & 2671 & 10880 & 2657 & 10866 & $4.6 e+01$ & $2.6 e+00$ & $1.059302 \mathrm{e}+09$ & 1 & 8 & 32.32 \\
\hline bgprtr & 20 & 40 & 20 & 40 & $1.9 e-01$ & $3.3 e-01$ & $8.008869 e+06$ & 0 & 6 & 0.01 \\
\hline box1 & 231 & 261 & 231 & 261 & $5.9 \mathrm{e}-02$ & $1.0 e+00$ & $5.775809 \mathrm{e}+02$ & 0 & 4 & 0.05 \\
\hline ceria3d & 3576 & 4400 & 3576 & 4400 & $8.0 \mathrm{e}-02$ & $7.4 e-02$ & $-9.975419 e-01$ & 1 & 12 & 13.60 \\
\hline chemcom & 288 & 744 & 288 & 744 & $4.9 e+02$ & $1.9 e+00$ & $3.908033 e+05$ & 0 & 8 & 0.21 \\
\hline cplex1 & 3005 & 5224 & 3005 & 5224 & $5.0 e+07$ & $9.2 e+00$ & $-2,701093 e+09$ & 0 & 5 & 1.09 \\
\hline cplex2* & 224 & 378 & 224 & 378 & $3.1 \mathrm{e}-06$ & $8.4 e-06$ & $6.550750 \mathrm{e}-01$ & 0 & 35 & 0.49 \\
\hline $\operatorname{ex} 72 a$ & 197 & 215 & 197 & 215 & $4.2 \mathrm{e}-01$ & $1.0 e+00$ & $4.579770 \mathrm{e}+02$ & 0 & 4 & 0.03 \\
\hline $\operatorname{ex} 73 a$ & 193 & 211 & 193 & 211 & $4.1 \mathrm{e}-01$ & $1.0 \mathrm{e}+00$ & $4.449144 \mathrm{e}+02$ & 0 & 4 & 0.03 \\
\hline forest 6 & 66 & 131 & 66 & 131 & $9.2 \mathrm{e}+01$ & $6.5 e-01$ & $4.139797 e+05$ & 0 & 11 & 0.03 \\
\hline galenet & 8 & 14 & 5 & 9 & $4.7 e+01$ & $9.4 e-01$ & $0.000000 \mathrm{e}+00$ & 0 & 5 & 0.00 \\
\hline gosh & 3792 & 13455 & 3479 & 12502 & $1.7 e+01$ & $1.1 \mathrm{e}+01$ & $4.141377 \mathrm{e}+02$ & 1 & 13 & 31.34 \\
\hline $\operatorname{gran} t$ & 2658 & 2525 & & & & & & & & \\
\hline greenbea-i & 2393 & 5596 & 1933 & 4153 & $1.1 \mathrm{e}+04$ & $7.7 e+00$ & $2.433071 \mathrm{e}+03$ & 0 & 10 & 3.87 \\
\hline itest 2 & 9 & 13 & 9 & 13 & $2.0 e+01$ & $4.6 \mathrm{e}-01$ & $0.000000 e+00$ & 0 & 5 & 0.00 \\
\hline itest6 & 11 & 17 & 10 & 15 & $5.0 e+05$ & $1.1 \mathrm{e}+00$ & $8.730497 \mathrm{e}+05$ & 0 & 5 & 0.01 \\
\hline klein1 & 54 & 108 & 54 & 108 & $3.1 \mathrm{e}+03$ & $9.2 \mathrm{e}+00$ & $0.000000 \mathrm{e}+00$ & 0 & 32 & 0.22 \\
\hline klein2 & 477 & 531 & 477 & 531 & $3.0 e+04$ & $3.7 e+02$ & $0.000000 \mathrm{e}+00$ & 2 & 22 & 14.73 \\
\hline klein3* & 994 & 1082 & 994 & 1082 & $9.6 e+04$ & $1.4 e+03$ & $0.000000 \mathrm{e}+00$ & 2 & 31 & 157.42 \\
\hline mondou2 & 312 & 604 & 259 & 467 & $8.0 e+00$ & $4.0 e+00$ & $6.313890 \mathrm{e}+08$ & 0 & 8 & 0.11 \\
\hline pang & 361 & 741 & 333 & 685 & $1.1 \mathrm{e}-02$ & $3.6 \mathrm{e}+00$ & $2.108127 e+04$ & 0 & 28 & 0.82 \\
\hline pilot $4 \mathrm{i}$ & 410 & 1123 & 396 & 1022 & $1.5 e+04$ & $5.7 e-01$ & $-1.377993 e+03$ & 1 & 33 & 3.50 \\
\hline qual & 323 & 464 & 305 & 441 & $2.2 \mathrm{e}+00$ & $4.8 \mathrm{e}-03$ & $-5.835719 e+04$ & 0 & 35 & 0.82 \\
\hline reactor & 318 & 808 & 269 & 602 & $9.9 e+00$ & $6.4 \mathrm{e}-01$ & $-3.285658 \mathrm{e}+05$ & 0 & 9 & 0.23 \\
\hline refinery & 323 & 464 & 303 & 439 & $3.5 e+01$ & $6.4 e-01$ & $-5.227856 e+04$ & 0 & 20 & 0.53 \\
\hline vol1 & 323 & 464 & 305 & 441 & $2.7 e+00$ & $5.9 e-02$ & $-6.099720 e+04$ & 0 & 31 & 0.73 \\
\hline woodinfe ${ }^{\dagger}$ & 35 & 89 & & & & & & & & \\
\hline
\end{tabular}

Table 3: NEMS Problems

\begin{tabular}{|c|c|c|c|c|c|c|c|c|c|c|}
\hline \multirow[b]{2}{*}{ Name } & \multicolumn{2}{|c|}{$\begin{array}{c}\text { Before } \\
\text { Preprocessing }\end{array}$} & \multicolumn{2}{|c|}{$\begin{array}{c}\text { After } \\
\text { Preprocessing }\end{array}$} & \multirow{2}{*}{$\begin{array}{l}\text { Relative } \\
\text { Infeas }\end{array}$} & \multirow{2}{*}{$\begin{array}{c}\text { Relative } \\
\text { Compl }\end{array}$} & \multirow{2}{*}{$\begin{array}{c}\text { Primal } \\
\text { Objective }\end{array}$} & \multirow{2}{*}{$\begin{array}{l}\text { Max } \\
\text { Add. } \\
\text { Corr. }\end{array}$} & \multirow[b]{2}{*}{ Iters } & \multirow{2}{*}{$\begin{array}{l}\text { CPU } \\
\text { Time } \\
\text { [secs] }\end{array}$} \\
\hline & Rows & Cols & Rows & Cols & & & & & & \\
\hline NEMSafm & 334 & 2348 & 322 & 1402 & $1.2 \mathrm{e}-14$ & $1.7 \mathrm{e}-11$ & $-6.792374 e+03$ & 0 & 17 & 0.47 \\
\hline NEMScem & 651 & 1712 & 479 & 1540 & $3.3 e-10$ & $1.9 \mathrm{e}-08$ & $8.977233 e+04$ & 0 & 19 & 0.75 \\
\hline NEMSemm1 & 3945 & 75352 & 3230 & 41048 & $4.9 \mathrm{e}-15$ & $3.6 \mathrm{e}-06$ & $5.129614 \mathrm{e}+05$ & 1 & 64 & 516.40 \\
\hline NEMSemm2 & 6943 & 48878 & 4526 & 26754 & $1.6 \mathrm{e}-10$ & $2.2 \mathrm{e}-06$ & $5.810806 \mathrm{e}+05$ & 0 & 37 & 35.52 \\
\hline NEMSpmm1 $1_{\max }$ & 2372 & 8903 & 2227 & 7145 & $2.4 \mathrm{e}-08$ & $4.8 \mathrm{e}-08$ & $3.274158 \mathrm{e}+05$ & 1 & 38 & 32.48 \\
\hline NEMSpmm $2_{\max }$ & 2301 & 8734 & 2081 & 7944 & $3.0 \mathrm{e}-08$ & $1.9 e-07$ & $2.917948 \mathrm{e}+05$ & 1 & 40 & 38.86 \\
\hline NEMSwrld $_{\max }$ & 7138 & 28550 & 5621 & 23706 & $7.3 e-13$ & $1.7 e-06$ & $-2.603093 e+02$ & 2 & 41 & 546.08 \\
\hline
\end{tabular}




\section{Acknowledgments}

We acknowledge the contribution of Marc Wenzel, who programmed the dense-column-handling and conjugate gradient refinement features that were added for the beta-2.0 release. We are grateful also to Doug Moore for various advice and for pointing out and repairing many memory leaks in the beta-1.0 release, and to Hans Mittelmann for running many of the benchmark tests and for compiling on various architectures, including DEC Alpha and Linux. Thanks also to Erling Andersen for helpful advice and comments.

\section{References}

[1] E. D. Andersen And K. D. Andersen, Presolving in linear programming, Mathematical Programming, 71 (1995), pp. 221-245.

[2] A. R. CuRTIS AND J. K. REID, On the automatic scaling of matrices for Gaussian elimination, J. Inst. Maths Applics, 10 (1972), pp. 118-124.

[3] J. R. Gilbert, E. NG, And B. W. Peyton, An efficient algorithm to compute row and column counts for sparse cholesky factorization, SIAM Journal on Matrix Analysis and Applications, 15 (1994), pp. 1075-1091.

[4] J. Gondzio, Multiple centrality corrections in a primal-dual method for linear programming, Computational Optimization and Applications, 6 (1996), pp. 137-156.

[5] J. W.-H. LIU, Modification of the minimum degree algorithm by multiple elimination, ACM Transactions on Mathematical Software, 11 (1985), pp. 141-153.

[6] - The role of elimination trees in sparse factorization, SIAM Journal on Matrix Analysis and Applications, 11 (1990), pp. 134-172.

[7] S. MEHRotra, On the implementation of a primal-dual interior point method, SIAM Journal on Optimization, 2 (1992), pp. 575-601.

[8] E. NG AND B. W. PEYTON, Block sparse Cholesky algorithms on advanced uniprocessor computers, SIAM Journal on Scientific Computing, 14 (1993), pp. 1034-1056.

[9] S. J. WRIGHT, The Cholesky factorization in interior-point and barrier methods, Preprint MCS-P600-0596, Mathematics and Computer Science Division, Argonne National Laboratory, Argonne, ll., May 1996.

[10] _- Primal-Dual Interior-Point Methods, SIAM Publications, Philadelphia, Pa., 1997.

[11] Y. ZHANG, User's Guide to LIPSOL, Department of Mathematics and Statistics, University of Maryland Baltimore County, Baltimore, Md., July 1995.

[12] _ Solving large-scale linear programs by interior-point methods under the MATLAB enviroment, Technical Report TR96-01, Department of Mathematics and Statistics, University of Maryland Baltimore County, Baltimore, Md., 1996.

[13] Annual Energy Outlook 1996, Energy Information Administration, U. S. Department of Energy, Washington, DC 20585, 1996. Document DOE/EIA-0383(96). 